\title{
THE COMPLETION OF AN ABELIAN CATEGORY
}

\author{
BY
}

\section{H. B. STAUFFER}

ABSTRACT. Any category $\underline{A}$ can be embedded in its right completion $\hat{A}$. When $\underline{A}$ is small and abelian, this completion $\underline{A}$ is AB5 and the embedding is exact.

1. Introduction. Given a category $\underline{A}$, we can construct its completion $\underline{\hat{A}}$ with respect to direct limits. $\underline{\hat{A}}$ is a free completion of $\underline{A}$, for $\underline{A}$ can be embedded in $\underline{\hat{A}}$, and any functor $F: \underline{A} \rightarrow \underline{B}$, where $\underline{B}$ is closed under direct limits, allows a unique extensi on $\hat{F}: \underline{A} \rightarrow \underline{B}$. This construction is well known. However, we shall show, in addition, that if $\underline{A}$ is small and abelian, $\underline{\hat{A}}$ is an AB5 category with a generator, and the embedding is exact. Hence, $\underline{A}$ has enough injectives. In fact, $\underline{\hat{A}}$ is equivalent to Sex $(\underline{A} \underline{\mathrm{op}}, \underline{A b})$, the category of all left exact functors from the dual category of $\underline{A}$ to the category of abelian groups. Let us note some terminology and conventions which will be observed. If $\underline{A}$ is a category, $|\underline{A}|$ will denote the class of objects in $\underline{A}$. $\operatorname{Hom}_{A}(A, B)$ will be written $\underline{A}(A, B)$. All functors will be covariant, unless stated othērwise, and additive, whenever meaningful. If $\underline{A}$ and $\underline{B}$ are categories, $\underline{B}-$ will denote the category of functors from $\underline{A}$ to $\underline{B}$ and natural transformations between them. Direct limit, often denoted by $\underline{\lim }$, will be indexed by any category, whereas directed direct limit will be indexed by a directed set. The dual concepts are inverse limit and directed inverse limit, respectively. If a category $\underline{A}$ has direct limits for all functors $F: \underline{D} \rightarrow \underline{A}$ where $\underline{D}$ is a small category, then $\underline{A}$ is right complete; the special case where $\underline{A}$ has directed limits for all functors $F: I \rightarrow \underline{A}$ where $I$ is a directed set is called closed under directed direct limits. The dual concepts are left complete and closed under directed inverse limits, respectively.

This paper owes a debt to Saul Lubkin, who initially suggested many of the results to me. I wish also to thank Professor Saunders Mac Lane for his many helpful suggestions and thoughtful encouragement during the preparation of this paper.

2. Given a category $\underline{A}$, we want to construct the category $\underline{\hat{A}}$ in a manner dual to the category $\underline{A}^{\mathrm{pro}}$ as introduced by Grothendieck (cf. [8, pp. 1-4]). Let us recall his procedure.

Received by the editors February 24, 1970.

AMS 1970 subject classifications. Primary 18A35; Secondary 18E20, 18E10.

Key words and phrases. Right completion of a category, embedding theorem, $\mathrm{AB} 5$ category, left exact functors, adjoint functors. 
To each object $A \in|\underline{A}|$ one can associate the representable functor $b_{A}=$ $\underline{A}(, A): \underline{A}^{\mathrm{op}} \rightarrow \underline{\text { Ens }}$, the category of sets. For each morphism $A \rightarrow A^{\prime}$ in $\underline{A}$, one clearly obtains a morphism $b_{A} \rightarrow b_{A}$ ' of functors. $b_{A}$ is a functor covariant in $A$, i.e. one has a functor $b: \underline{A} \rightarrow \underline{\text { Ens }}{ }^{A}{ }^{\text {op }}$. Using the Yoneda Lemma (cf. [13, pp. 97-99]), we find that the natural map

$$
\underline{A}\left(A, A^{\prime}\right) \rightarrow \underline{\text { Ens }}^{\underline{A}^{\mathrm{op}}\left(b_{A}, b_{A^{\prime}}\right) .}
$$

is a bijection. If a functor $F \in\left|\underline{\text { Ens }}{ }^{A}{ }^{\circ \mathrm{p}}\right|$ is naturally isomorphic to a functor of the form $b_{A}$, then $F$ is said to be representable. Thus $b$ defines an equivalence between the category $\underline{A}$ and the full subcategory of Ens ${ }^{A}{ }^{\circ \mathrm{p}}$ consisting of representable functors.

Let us extend this procedure. To each functor $A_{.}: I \rightarrow \underline{A}$ where $I$ is any directed set regarded as a category (cf. [12, p. 43]), we associate the functor $b_{A}=$

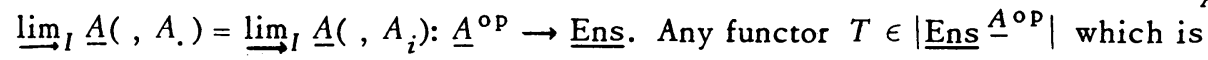
naturally isomorphic to one of the form $b_{A}$. will be called ind-representable. Consider another such functor $B_{;}: J \rightarrow \underline{A}$ and its associat ed functor $b_{B}$. Again using the Yoneda Lemma, we have a bijection

$$
\begin{aligned}
& \left.\left.\underline{\text { Ens }}^{\underline{A}^{\mathrm{OP}}\left(b_{A} \cdot{ }^{b} B\right.} \cdot\right)=\underline{\mathrm{Ens}}^{\underline{A}} \stackrel{{ }^{\mathrm{OP}}}{\left(\lim _{I} I\right.} \underline{A}\left(, A_{i}\right), \underline{\lim } J \underline{A}\left(, B_{j}\right)\right)
\end{aligned}
$$

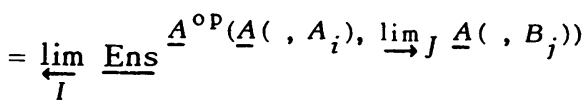

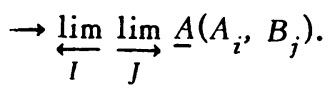

With this in mind, we define the category $\underline{A}$ of ind-objects of $\underline{A}$. Its objects will consist of functors $A_{.}: I \rightarrow \underline{A}$ where $I$ is any directed set, and, if $A .: I \rightarrow \underline{A}$ and $B .: J \rightarrow \underline{A}$ are two such objects, we let

$$
\underline{\hat{A}}\left(A_{,}, B_{.}\right)=\underset{I}{\lim } \underset{J}{\lim } A\left(A_{i}, B_{j}\right) .
$$

We shall show that there is an evident composition of morphisms in $\underline{A}$ satisfying the category axioms. Then, $A . \longrightarrow \underline{\lim }_{I} \underline{A}(, A$.) defines a functor $H$ which establishes an equivalence between the category $\underline{A}$ of ind-objects of $\underline{A}$ and the category of ind-representable functors in Ens ${ }^{A}{ }^{\circ}$.

Let us examine the morphisms of $\underline{\hat{A}}$ more closely. An element of $\stackrel{\lim }{\longrightarrow} J \underline{A}\left(A_{i}, B_{j}\right)$ consists of an index $j$ plus a morphism $f_{i}: A_{i} \rightarrow B_{j}$ in $\underline{A}$ with the following equivalence relation. $f_{i}: A_{i} \rightarrow B_{j}$ and $f_{i}^{\prime}: A_{i} \rightarrow B_{j}^{\prime}$ are equivalent if and only if there exists $j^{\prime \prime} \geq j, j^{\prime}$ in $J$ such that the following diagram commutes: 


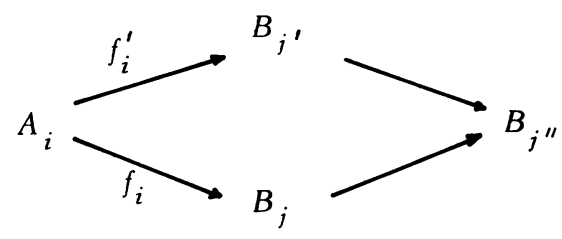

Hence given objects $A_{.}: I \rightarrow \underline{A}$ and $B .: J \rightarrow \underline{A}$ in $\underline{\hat{A}}$, an element of $\underline{\hat{A}}\left(A_{.}, B_{.}\right)=$

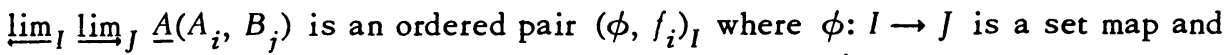
$f_{i}: A_{i} \rightarrow B_{\phi(i)}$ is a morphism in $\underline{A}$ for each $i \in I$. If $i^{\prime} \geq i$ in $I$, there exists $j \geq$ $\phi(i), \phi\left(i^{\prime}\right)$ in $J$ such that the following diagram commutes:

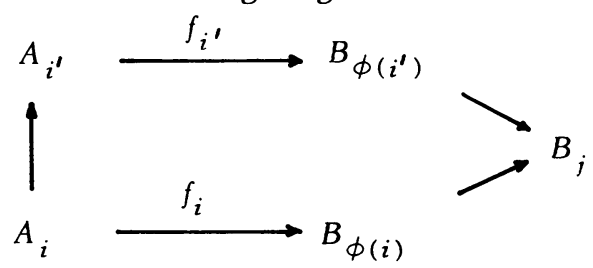

An equivalence relation exists. In $\underline{\hat{A}}\left(A, B_{.}\right),\left(\phi, f_{i}\right)_{I} \sim\left(\phi^{\prime}, f_{i}^{\prime}\right)_{I}$ if and only if for each $i \in I$ there exists $j \geq \phi(i), \phi^{\prime}(i)$ in $J$ such that the following diagram commutes:

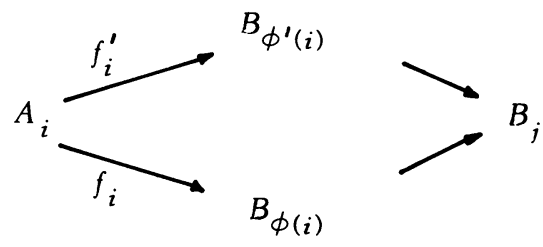

It is now immediate that $\underline{A}$ is indeed a category. We shall often refer to a morphism $\left(\phi, f_{i}\right)_{I}$ as $(\phi, f)$ or just $f$ and to an object $A_{.}: I \rightarrow \underline{A}_{\text {as }}\left\{A_{i}\right\}_{I},\{A\}_{I}$, or $\left\{A_{i}, \alpha\right\}_{I}$ (cf. [13, pp. 47-48]). If $A \in|\underline{A}|,\{A\}$ will denote the functor $A .: P \rightarrow \underline{A}$ where $P$ is the one point directed set and $A$. is defined by $\rightarrow \rightarrow A$.

3. When a finite number of morphisms in $\underline{\hat{A}}$ are under consideration they may be regarded as natural transformations of functors from a common directed set. Let us be more precise. Recall that a directed set $I$ is pointwise finitely preceded (p.f.p.) if and only if, for each $i_{0} \in I$, the subset $\left\{i \mid i \leq i_{0}\right.$ in $\left.I\right\}$ is finite (cf. [14]).

Proposition 3.1. Let $D$ be the finite diagram in $\underline{\hat{A}}$ consisting of $n$ objects $\left\{A^{1}\right\}_{I_{1}},\left\{A^{2}\right\}_{I_{2}}, \cdots,\left\{A^{n}\right\}_{I_{n}}$ in $\underline{\hat{A}}$ and, for $1 \leq b<k \leq n$, a finite set $M_{b, k} \subset$ $\underline{\hat{A}}\left(\left\{A^{b}\right\}_{I_{b}},\left\{A^{k}\right\}_{I_{k}}\right)$. Then there exists a p.f.p. directed set $I$ and a finite diagram $D^{\prime}$ in the category $\underline{A}^{I} \rightarrow \underline{\hat{A}}$ consisting of $n$ objects $\left\{B^{1}\right\}_{I},\left\{B^{2}\right\}_{I}, \ldots,\left\{B^{n}\right\}_{I}$ in $\underline{A}^{I}$ and, for $1 \leq b<k \leq n$, a finite set $M_{b, k}^{\prime} \subset \underline{A}^{I}\left(\left\{B^{b}\right\}_{I},\left\{B^{k}\right\}_{I}\right)$ such that $D^{\prime}$ is equivalent to $D$ in $\underline{\hat{A}}$, i.e. there exist isomorphisms $i^{r}:\left\{B^{r}\right\}_{I} \rightarrow\left\{A^{r}\right\}_{I_{r}}$ in $\underline{\hat{A}}, 1 \leq r \leq n$, such that $M_{b, k}^{\prime}=\left(i^{k}\right)^{-1} \circ M_{b, k} \circ i^{b}$. 
Proof. Let us proceed with several preliminary lemmas. Let $\left(\phi, f_{i}\right)_{I}:\left\{A_{i}, \alpha\right\}_{I}$ $\rightarrow\left\{B_{i}, \beta\right\}_{J}$ be a morphism in $\underline{\hat{A}}$. We shall need to consider a new morphism $\left(\rho, g_{i}\right)_{I}:\left\{A_{i}, \alpha\right\}_{I} \rightarrow\left\{B_{j}, \beta\right\}_{J}$ constructed from $\left(\phi, f_{i}\right)_{I}$ in the following way. For each $i \in I$, pick some $j \geq \phi(i)$ in $J$, and let $\rho(i)=j$. Define $g_{i}=\beta_{\phi(i)}^{j} \circ f_{i}$.

Lemma 3.2. With the above notation, $\left(\rho, g_{i}\right)_{I}$ is a morphism in $\underline{\hat{A}}$. Furthermore, $\left(\rho, g_{i}\right)_{l} \sim\left(\phi, f_{i}\right)_{l}$.

Lemma 3.3. Let $A_{.}=\left\{A_{i}, \alpha\right\}_{I} \in|\underline{\hat{A}}|$, and let $F: I^{\prime} \rightarrow I$ be a cofinal functor between directed sets. Then, if $\left\{A_{F\left(i^{\prime}\right)}, \alpha\right\}_{I^{\prime}}=A \circ F: I^{\prime} \rightarrow \underline{A}$, the morphism $\left(F, \mathrm{id}_{F\left(i^{\prime}\right)}\right)_{I^{\prime}}:\left\{A_{F\left(i^{\prime}\right)}, \alpha\right\}_{I^{\prime}} \rightarrow\left\{A_{i}, \alpha\right\}_{I}$ defines an isomorphism in $\hat{A}$, where id is the identity map.

Proof. Use the fact that $F$ is cofinal to define the set map $\rho: I \rightarrow I^{\prime}$ as follows. For each $i \in I$, choose $\rho(i)=i^{\prime} \in I^{\prime}$ such that $F\left(i^{\prime}\right) \geq i$ in $I$. Then $\left(\rho, \alpha_{i}^{F(\rho(i))}\right)_{I^{\prime}}:\left\{A_{i}, \alpha\right\}_{I} \rightarrow\left\{A_{F\left(i^{\prime}\right)}, \alpha\right\}_{I^{\prime}}$ gives us the inverse of $\left(F, \mathrm{id}_{F\left(i^{\prime}\right)}\right)_{I^{\prime}}$. Q.E.D.

Given a morphism $\left(\phi, f_{i}\right)_{I}:\left\{A_{i}, \alpha\right\}_{I} \rightarrow\left\{B_{j}, \beta\right\}_{J}$ in $\underline{\hat{A}}$, we can always consider the range of $\phi$ to be cofinal in $J$. More precisely,

Lemma 3.4. If $\left(\phi, f_{i}\right)_{I}:\left\{A_{i}, \alpha\right\}_{I} \rightarrow\left\{B_{j}, \beta\right\}_{J}$ is a morphism in $\underline{\hat{A}}$, then there exist an isomorphism b: $\left\{C_{k}, \gamma\right\}_{K} \rightarrow\left\{A_{i}, \alpha\right\}_{I}$ and a morphism $\left(\rho, g_{k}\right)_{K}:\left\{C_{k}, \gamma\right\}_{K} \rightarrow$ $\left\{B_{j}, \beta\right\}_{J}$ such that

(i) $\left(\rho, g_{k}\right)_{K} \sim\left(\phi, f_{i}\right)_{I} \circ b$, and

(ii) the range of $\rho$ is cofinal in $J$.

Proof. If the range of $\phi$ is already cofinal in $J$, we are done. If not, there exists $j_{0} \in J$ such that $\phi(i) \geq j_{0}$ does not hold for any $i \in I$. Let $J^{\prime}=\left\{j^{\prime} \mid j^{\prime} \geq j_{0}\right.$ in $J\}$ be the cofinal subset in $J$, and let $K=I \dot{\cup} J^{\prime}$, the disjoint union, with the following induced order. Use the original order for elements in $I$. Let $j^{\prime} \leq j^{\prime \prime} \leq j^{\prime}$ for all $j^{\prime}, j^{\prime \prime} \in J^{\prime}$. Finally, fix some $i_{0} \in I$, and, for each $j^{\prime} \in J^{\prime}, i \in I$, let $j^{\prime} \leq i$ or $j^{\prime} \geq i$ if and only if $i_{0} \leq i$ or $i_{0} \geq i$, respectively. $K$ is then a directed set. Define the order-preserving function $F: K \rightarrow I$ by letting $F(i)=i, i \in I$, and $F\left(j^{\prime}\right)$ $=i_{0}, j^{\prime} \in J^{\prime}$. Then $F$ is a cofinal functor, and, by Lemma 3.3, there exists an isomorphism $b=\left(F, \mathrm{id}_{F(k)}\right)_{K}:\left\{C_{k}, y\right\}_{K} \rightarrow\left\{A_{i}, a\right\}_{I}$ where $\left\{C_{k}, y\right\}_{K}=A \circ$ $F: K \rightarrow \underline{A}$ and $\left\{A_{i}, \alpha\right\}_{I}=A: I \rightarrow A$. Then, we use Lemma 3.2. to construct the morphism $\left(\rho, g_{k}\right)_{K} \sim\left(\phi, f_{i}\right)_{I} \circ b$ as follows. For each $i \in I$, let $\rho(i)=\phi(i), g_{i}=f_{i}$, and, for each $j^{\prime} \in J^{\prime}$, let $\rho\left(j^{\prime}\right)=j$, where $j \geq j^{\prime}, \phi\left(i_{0}\right)$ in $J, g_{j^{\prime}}=\beta_{\phi\left(i_{0}\right)}^{j} \circ f_{i_{0}}$. Then the image of $\rho$ is cofinal in $J^{\prime}$, hence in $J$. Q.E.D.

Lemma 3.5. Let $\left(\phi, f_{i}\right)_{I}:\left\{A_{i}, \alpha\right\}_{I} \rightarrow\left\{B_{j}, \beta\right\}_{J}$ be a morphism with $I$ a p.f.p. directed set. Then there exists a morphism $\left(\rho, g_{i}\right)_{I}:\left\{A_{i}, \alpha\right\}_{I} \rightarrow\left\{B_{j}, \beta\right\}_{J}$ such that (i) $\left.\rho(i) \geq \phi^{\prime}, i\right)$ in $J$ and $g_{i}=\beta_{\phi(i)}^{\rho(i)} \circ f_{i}$ for all $i \in I$ (bence $\left(\rho, g_{i}\right) \sim\left(\phi, f_{i}\right)$, and, if the image of $\phi$ is cofinal in $J$, the image of $\rho$ is also cofinal in $J$ ), and

(ii) if $i \leq i^{\prime}$ in $I$, then $\rho(i) \leq \rho\left(i^{\prime}\right)$ in $J$ and the following diagram commutes: 


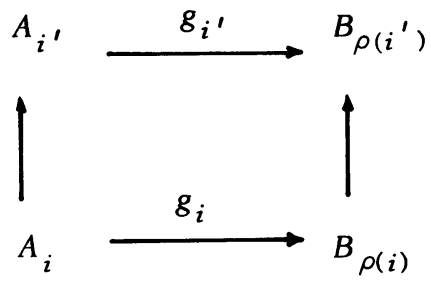

Proof. We use transfinite induction to define $\rho$ and $g_{i}$ on $I$. Let $<^{+}$wellorder the set $I$. Let $i_{0} \in I$, and suppose $\rho$ and $g_{i}$ have been defined satisfying (i) and (ii) on $I_{0}=\left\{i \mid i \leq i^{\prime}, i^{\prime}<{ }^{+} i_{0}\right.$ in $\left.l\right\}$. Then, letting $I_{0}^{\prime}=\left\{i \mid i \leq i_{0}\right.$ in $\left.I\right\}$, extend $\rho$ and $g_{i}$ to $I_{0}^{\prime \prime}=I_{0} \cup I_{0}^{\prime}$ by defining $\rho$ and $g_{i}$ on $I_{0}^{\prime \prime}-I_{0}$ so that (i) and (ii) are satisfied on $I_{0}^{\prime}$. This is possible since $I$ is a p.f.p. directed set $\ldots$ we can define $\rho$ to be an appropriate constant on the finite set $I_{0}^{\prime \prime}-I_{0}$. Then it is immediate that $\rho$ and $g_{i}$ satisfy (i) and (ii) on $I_{0}^{\prime \prime}$. We have extended $\rho$ and $g_{i}$ to include $i_{0}$. Hence, by transfinite induction, we can define $\rho$ and $g_{i}$ satisfying (i) and (ii) on all of $I$. Q.E.D.

Now we are ready to prove Proposition 3.1. We proceed by induction on $n$. If $n=1$, there exist a p.f.p. directed set $I$ and cofinal functor $F: I \rightarrow I_{1}$ (cf. [14, p. 375]). Then, by Lemma 3.3, there exists an isomorphism $i^{1}:\left\{B^{1}\right\}_{I} \rightarrow\left\{A^{1}\right\}_{I_{1}}$ where $\left\{B^{1}\right\}_{I}=A^{1} \circ F: I \rightarrow \underline{A}$ and $\left\{A^{1}\right\}_{I_{1}}=A_{.}^{1}: I_{1} \rightarrow \underline{A}$. Hence, the proposition is true for $n=1$. Let $n>1$, and assume the proposition holds for the case $n-1$. Then there exist a p.f.p. directed set $J$, isomorphisms $e^{r}:\left\{C^{r}\right\}_{J} \rightarrow\left\{A^{r}\right\}_{I_{r}}$ in $\underline{\hat{A}}, 2 \leq r \leq$ $n$, and morphisms $M_{b, k}^{\prime \prime}=\left(e^{k}\right)^{-1} \circ M_{b, k} \circ e^{b} \subset \underline{\hat{A}}\left(\left\{C^{b}\right\}_{J},\left\{C^{k}\right\}_{J}\right), 2 \leq b<k \leq n$, such that

(i) all $\left\{C^{r}\right\}_{J}$ are indexed by $J, 2 \leq r \leq n$, and

(ii) each morphism in $M_{b, k}^{\prime \prime}$ is of the form (id, $g$ ) where $g$ is a natural transformation of functors.

Suppose there are no morphisms with domain $\left\{A^{1}\right\}_{I_{1}}$. Then using Lemma 3.3, we can construct an isomorphism $e^{1}:\left\{C^{1}\right\}_{K} \rightarrow\left\{A^{1}\right\}_{I_{1}}$ with $K$ a p.f.p. directed set. Let $I$ be the product of $K$ and $J$ in the category of directed sets and functors between them, i.e. $I=(k, j), k \in K, j \in J$, and $(k, j) \leq\left(k^{\prime}, j^{\prime}\right)$ in $I$ if and only if $k \leq k^{\prime}$ in $K$ and $j \leq j^{\prime}$ in $J$. Then $I$ is a p.f.p. directed set, and we have the cofinal projection functors $\pi_{1}: I \rightarrow K$ and $\pi_{2}: I \rightarrow J$ defined by

$$
\pi_{1}(k, j)=k, \quad \pi_{2}(k, j)=j \text {. }
$$

Hence, using Lemma 3.3, we have the isomorphisms $b^{1}:\left\{B^{1}\right\}_{I} \rightarrow\left\{C^{1}\right\}_{K}$ and $b^{r}$ : $\left\{B^{r}\right\}_{I} \rightarrow\left\{C^{r}\right\}_{J}, 2 \leq r \leq n$, where $\left\{B^{1}\right\}_{I}=C^{1} \circ \pi_{1}: I \rightarrow \underline{A},\left\{C^{1}\right\}_{K}=C_{.}^{1}: K \rightarrow \underline{A}$, $\left\{B^{r}\right\}_{I}=C^{r} \circ \pi_{2}: I \rightarrow \underline{A}$, and $\left\{C^{r}\right\}_{J}=C^{r}: J \rightarrow A$. Then we can let $i^{r}=e^{r} \circ b^{r}, 1 \leq$ $r \leq n$, and the proposition is proved.

Suppose there are $k$ morphisms $\left(\phi^{s}, f^{s}\right):\left\{A^{1}\right\}_{I_{1}} \rightarrow\left\{A^{r(s)}\right\}_{\left.I_{r(s)}\right)}, 1 \leq s \leq k, 2 \leq$ $r(s) \leq n$. Then we can use Lemma 3.2 to obtain morphisms $\left(\rho, b^{s}\right) \sim\left(e^{r(s)}\right)^{-1} \circ$ 
$\left(\phi^{s}, f^{s}\right), 1 \leq s \leq k$, all having the same set function $\rho$, i.e. for each $i_{1} \in I_{1}$, choose $\rho\left(i_{1}\right) \geq \phi^{s}\left(i_{1}\right), 1 \leq s \leq k$, and define $b_{i_{1}}^{s}$ as in the the lemma. Next we can use Lemma 3.4 to obtain an isomorphism $e^{1}:\left\{C^{1}\right\}_{K} \rightarrow\left\{A^{1}\right\}_{I_{1}}$ and morphisms $\left(\theta, m^{s}\right) \sim$ $\left(\rho, b^{s}\right) \circ e^{1}, 1 \leq s \leq k$, such that the image of $\theta$ is cofinal in $J$. Next we can obtain a cofinal functor $F: I \rightarrow K$ where $F:|I| \rightarrow|K|$ is onto as a set function and $I$ is a p.f.p. directed set. Then, using Lemma 3.3, we obtain an isomorphism $b^{1}$ : $\left\{B^{1}\right\}_{I} \rightarrow\left\{C^{1}\right\}_{K}$ where $I$ is a p.f.p. directed set and the image of the set map of the compositions $\left(\theta, m^{s}\right) \circ b^{1}$ remains cofinal in $J$. Hence, we can obtain morphisms $\left(\sigma, n^{s}\right) \sim\left(\theta, m^{s}\right) \circ b^{1}, 1 \leq s \leq k$, satisfying (i) and (ii) of Lemma 3.5. Since the image of $\sigma$ is cofinal in $J$, we can use Lemma 3.3 to obtain isomorphisms $b^{r}$ : $\left\{B^{r}\right\}_{I} \rightarrow\left\{C^{r}\right\}_{J}, 2 \leq r \leq n$, and factorizations $\left(\sigma, n^{s}\right)=b^{r(s)} \circ\left(\mathrm{id}, g^{s}\right)$. Setting $i^{r}=$ $e^{r} \circ b^{r}, 1 \leq r \leq n$, we have our result. Q.E.D.

If $\underline{A}$ is abelian, any monomorphism (epimorphism) in $\underline{A}$ can be expressed in the form (id, $\left.b_{i}\right)_{I}$ where $b$ is a natural transformation of functors and each $b_{i}$ is a monomorphism (epimorphism) in $\underline{A}$. More precisely,

Corollary 3.6. Let $(\phi, f):\left\{A^{1}\right\}_{I_{1}} \rightarrow\left\{A^{2}\right\}_{I_{2}}$ be a monomorphism (epimorphism) in $\underline{\hat{A}}$ where $\underline{A}$ is abelian. Then there exist a directed set $I$, isomorphisms $i^{1}$ : $\left\{C^{1}\right\}_{I} \rightarrow\left\{A^{1}\right\}_{I_{1}}$ and $i^{2}:\left\{C^{2}\right\}_{I} \rightarrow\left\{A^{2}\right\}_{I_{2}}$ and a monomorphism (epimorphism) $\left(\mathrm{id}, b_{i}\right)_{I}:\left\{C^{1}\right\}_{I} \rightarrow\left\{C^{2}\right\}_{I}$ in $\underline{\hat{A}}$ yielding the commutative diagram

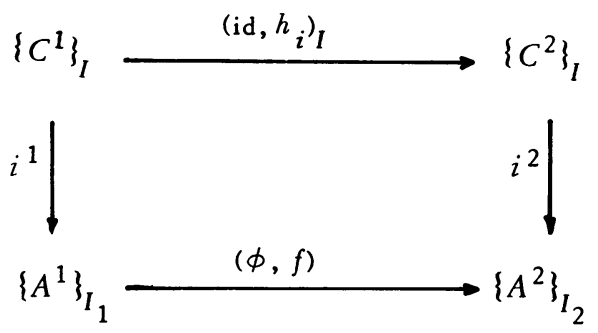

and satisfying

(i) $\left\{C^{1}\right\}_{1}$ and $\left\{C^{2}\right\}_{I}$ are both indexed by $I$,

(ii) $b: C_{.}^{1} \rightarrow C^{2}$ is a natural transformation of functors, and

(iii) each $b_{i}$ is a monomorphism (epimorphism) in $\underline{A}$.

Proof. From Proposition 3.1, there exist a directed set $I$, isomorphisms $e^{1}$ : $\left\{B_{i}^{1}, \beta^{1}\right\}_{I} \rightarrow\left\{A^{1}\right\}_{I_{1}}$ and $e^{2}:\left\{B_{i}^{2}, \beta^{2}\right\}_{I} \rightarrow\left\{A^{2}\right\}_{I_{2}}$, and a monomorphism (epimorphism) (id, $\left.g_{i}\right)_{I}:\left\{B_{i}^{1}, \beta^{1}\right\}_{I} \rightarrow\left\{B_{i}^{2}, \beta^{2}\right\}_{I}$ yielding the commutative diagram

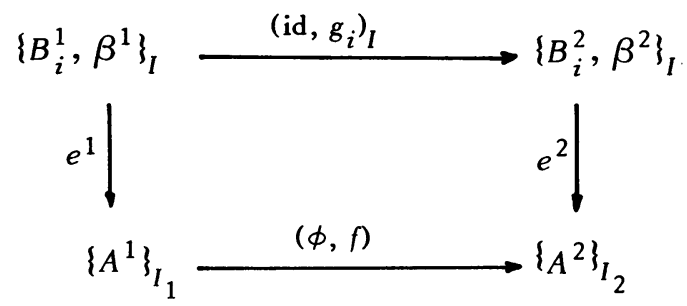


and satisfying

(i) $\left\{B_{i}^{1}, \beta^{1}\right\}_{I}$ and $\left\{B_{i}^{2}, \beta^{2}\right\}_{I}$ are both indexed by $I$, and

(ii) $g: B^{1} \rightarrow B^{2}$. is a natural transformation of functors.

Since $\underline{A}$ is abelian, for $i \leq i^{\prime}$ in $I$, the $g_{i}$ 's factor into the commutative diagram

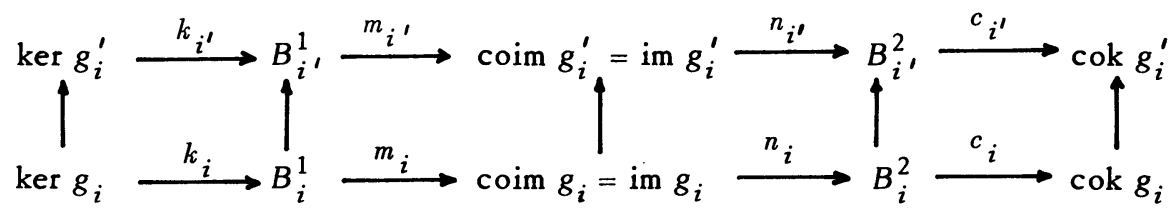

where the $m_{i}$ 's are epimorphisms and the $n_{i}$ 's are monomorphisms in $\underline{A}$. Thus in $\underline{\hat{A}}$ we have the morphisms

$$
\begin{aligned}
& \left\{\operatorname{ker} g_{i}\right\}_{I} \stackrel{\left(\mathrm{id}, k_{i}\right)_{I} \longrightarrow}{\longrightarrow}\left\{B_{i}^{1}, \beta^{1}\right\}_{I} \stackrel{\left(\mathrm{id}, m_{i}\right)_{I}}{\longrightarrow}\left\{\operatorname{coim} g_{i}\right\}_{I} \\
& =\left\{\mathrm{im} g_{i}\right\}_{I} \stackrel{\left(\mathrm{id}, n_{i}\right)_{I} \longrightarrow}{\longrightarrow}\left\{B_{i}^{2}, \beta^{2}\right\}_{I} \stackrel{\left(\mathrm{id}, c_{i}\right)_{I}}{\longrightarrow}\left\{\operatorname{cok} g_{i}\right\}_{I}
\end{aligned}
$$

where $\left(\mathrm{id}, g_{i}\right)_{I}=\left(\mathrm{id}, n_{i}\right)_{I} \circ\left(\mathrm{id}, m_{i}\right)_{I}$. If $\left(\mathrm{id}, g_{i}\right)_{I}$ is a monomorphism in $\underline{\hat{A}}$, then we claim (id, $\left.m_{i}\right)_{I}$ is an isomorphism in $\underline{\hat{A}}$. For, (id, $\left.g_{i}\right)_{l} \circ$ (id, $\left.k_{i}\right)_{I}=0$ implies

$\left(\mathrm{id}, k_{i}\right)_{I}=0$. This implies that, for each $i \in I$, there exists $\sigma(i)=i^{\prime} \geq i$ such that $\beta_{i}^{1} i^{\prime} \circ k_{i}=0$. Thus there exists a morphism $p_{i}$ yielding the following commutative diagram:

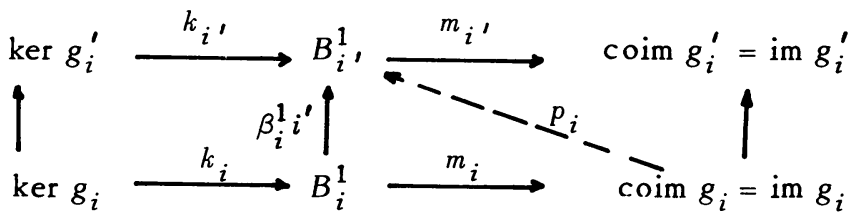

$\left(\sigma, p_{i}\right)_{I}$ is the inverse of (id, $\left.m_{i}\right)_{I}$. Letting $\left\{C^{1}\right\}_{I}=\left\{\operatorname{coim} g_{i}\right\}_{I},\left\{C^{2}\right\}_{I}=\left\{B_{i}^{2}, \beta^{2}\right\}_{I}$, $i^{1}=e^{1} \circ\left(\sigma, p_{i}\right)_{I}, i^{2}=e^{2}$, and $\left(\mathrm{id}, b_{i}\right)_{I}=\left(\mathrm{id}, n_{i}\right)_{I}$, we have our result. Similarly, if (id, $\left.g_{i}\right)_{I}$ is an epimorphism, then (id, $\left.n_{i}\right)_{I}$ is an isomorphism in $\hat{A}$. Let $\left\{C^{1}\right\}_{I}=$ $\left\{B_{i}^{1}, \beta^{1}\right\}_{I},\left\{C^{2}\right\}_{I}=\left\{\mathrm{im} g_{i}\right\}_{I}, i^{1}=e^{1}, i^{2}=e^{2} \circ\left(\mathrm{id}, n_{i}\right)_{I}$, and (id, $\left.b_{i}\right)_{I}=\left(\mathrm{id}, m_{i}\right)_{I}$, and we are done. Q.E.D.

4. Now we are ready for the main results about $\underline{\hat{A}}$.

Theorem 4.1. Let $\underline{A}$ be any category. Then

(i) $\underline{A}$ is closed under directed direct limits.

(ii) There exists a functor $I_{A}: \underline{A} \hookrightarrow \underline{\hat{A}}$ which is a full embedding, and, given a functor $F: \underline{A} \rightarrow \underline{B}$ where $\underline{B}$ is closed under directed direct limits, there exists a functor $\hat{F}: \underline{\hat{A}} \rightarrow \underline{B}$ which preserves directed direct limits and gives the commutative diagram: 


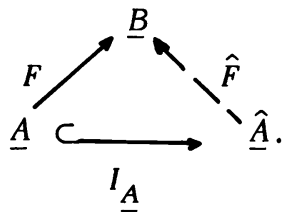

If $\eta: F \rightarrow G$ is a natural transformation between functors from $\underline{A}$ to $\underline{B}$, then there exists a unique natural transformation $\hat{\eta}: \hat{F} \rightarrow \hat{G}$ which extends $\eta$, i.e. if $A \in|\underline{A}|$, then $\hat{\eta}\left[I_{A}(A)\right]=\eta(A)$. Hence $\hat{F}$ is unique.

(iii) If $A$ is additive, it induces an additive structure on $\hat{A}$ such that $I_{\underline{A}}$ : $\underline{A}$ $\hookrightarrow \underline{A}$ is additive. If $F: \underline{A} \rightarrow \underline{B}$ is additive, then so is $\hat{F}: \underline{\hat{A}} \rightarrow \underline{B}$.

(iv) If $\underline{A}$ is abelian, $\underline{\hat{A}}$ is an $\mathrm{AB} 5$ category and $I_{A}: \underline{A} \hookrightarrow \underline{\hat{A}}$ is exact. If, in addition, $\underline{A}$ is small, then $\underline{\hat{A}}$ bas a generator and, bence, bas enough injectives.

Proof. Let $F: J \rightarrow \underline{A}$ be a functor where $J$ is a directed set. Let $F(j)=\left\{A_{i_{j}}^{j}, \alpha^{j}\right\}_{I_{j}}$, for each $j \in J$, and, for $j \leq j^{\prime}$ in $J$, let $F\left(j \leq j^{\prime}\right)=\left(\phi^{j, j^{\prime}}, f_{i_{j}}^{j_{j} j^{\prime}}\right)_{I_{j}}:\left\{A_{i_{j}}^{j}, \alpha^{j}\right\}_{I_{j}} \rightarrow$ $\left\{A_{i_{j}^{\prime}}^{j^{\prime}}, a^{j^{\prime}}\right\}_{I_{j}}$. Then we construct the direct limit of $F$ in $\underline{\hat{A}}$ as follows. Let $L=$ $\bigcup_{j \in J}^{i_{j}} I_{j}$ with the induced order $i_{j} \leq i_{j}^{\prime}$ if and only if $j \leq j^{\prime}$ in $J$ and $\phi^{j, j^{\prime}}\left(i_{j}\right) \leq i_{j}{ }^{\prime}$ in $I_{j}{ }^{\prime} . L$ is then a directed set. Then define the functor $D_{A}=L \rightarrow \underline{A}$ by letting $D_{i j}=A_{i j}^{j}, i_{j} \in I_{j}$, and $D\left(i_{j} \leq i_{j}{ }^{\prime}\right)=\left(\alpha^{j^{\prime}}\right)_{\phi^{j}, j^{\prime}\left(i_{j}\right)}^{i^{\prime}}{ }^{\prime} f_{i_{j}}^{j, j^{\prime}} . D \in|\underline{\hat{A}}|$ together with $\eta$ : $F \rightarrow$ const $D_{\text {. }}$, the natural transformation of functors defined by $\eta(j)=\left(\text { in, id } i_{j}\right)_{I_{j}}$ : $\left\{A_{i j}^{j}, \alpha^{j}\right\}_{I_{j}}=F(j) \rightarrow D_{\text {. }}$, where in is the inclusion map, give the desired direct limit of $F$ in $\underline{A}$.

$I_{\underline{A}}$ is defined by letting $I_{A}(A)=\{A\}, A \in|\underline{A}|$, and $I_{A}(f)=(\mathrm{id}, f)$ for each morphism $f$ in $\underline{A}$. Note that every object $A$. in $\underline{\hat{A}}$ is a directed direct limit of a functor which factors through $I_{\underline{A}}$, i.e.

$$
A_{.}=\left\{A_{i}, \alpha\right\}_{I}=\underset{I}{\lim }\left\{A_{i}\right\}=\underset{I}{\lim } I_{\underline{A}} \circ A_{.} \cdot
$$

Hence, since $\hat{F}$ preserves directed direct limits, we must have

$$
\hat{F}\left(A_{\bullet}\right)=\hat{F}\left(\underset{I}{\lim _{I}} I_{A} \circ A_{.}\right)=\underset{I}{\lim } \hat{F} \circ I^{A} \circ A_{.}=\underset{I}{\lim } F \circ A_{.}=\underset{I}{\lim } F\left(A_{i}\right),
$$

the directed direct limit in $\underline{B}$. Given a morphism $\left(\phi, f_{i}\right)_{I}:\left\{A_{i}, \alpha\right\}_{I} \rightarrow\left\{B_{j}, \beta\right\}_{J}$ in $\hat{A}, \hat{F}\left[\left(\phi, f_{i}\right)_{I}\right]: \underline{\lim }_{I} F\left(A_{i}\right) \rightarrow \varliminf_{J} F\left(B_{j}\right)$ is the map induced by the $F\left(f_{i}\right)$ on the direct limits in $\underline{B}$. Let $A .=\left\{A_{i}, \alpha\right\}_{l}$ be an object in $\underline{\hat{A}}$ and let $\bar{G}: \underline{\hat{A}} \rightarrow \underline{B}$ be any functor extending $G$. Define $\hat{\eta}\left[\left\{A_{i}, \alpha\right\}_{I}\right]: \hat{F}\left[\left\{A_{i}, a\right\}_{I}\right] \rightarrow \bar{G}\left[\left\{A_{i}, \alpha\right\}_{I}\right]$ as follows. For $i \leq i^{\prime}$ in $I$, we have the commutative diagram

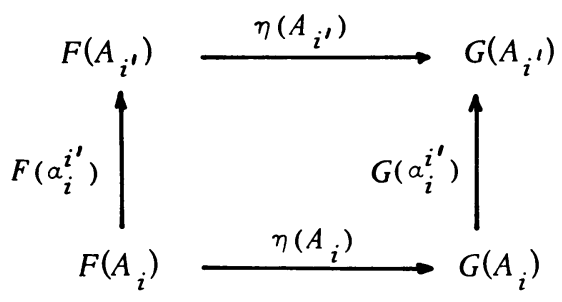


since $\eta$ is a natural transformation. Hence the $\eta\left(A_{i}\right)$ gives us a natural transformation of functors $F \circ A . \rightarrow G \circ A$. Taking the direct limit, we get an induced map $\hat{\eta}\left[\left\{A_{i}, \alpha\right\}_{I}\right]: \hat{F}\left[\left\{A_{i}, \alpha\right\}_{I}\right]=\underline{\lim }_{I} F \circ A . \rightarrow \bar{G}\left[\left\{A_{i}, \alpha\right\}_{I}\right]$. It is immediate that $\hat{\eta}$ extends $\eta$. Furthermore, since $\left\{A_{i}, \alpha\right\}_{I}=\underline{\lim }_{I}\left\{A_{i}\right\}$ in $\underline{\hat{A}}, \hat{\eta}$ is the unique map which extends $\eta$. Let $\left(\phi, f_{i}\right)_{I}:\left\{A_{i}, a\right\} \rightarrow\left\{B_{j}, \beta\right\}_{J}$ be a morphism in $\underline{\hat{A}}$. Then we have the commutative diagram

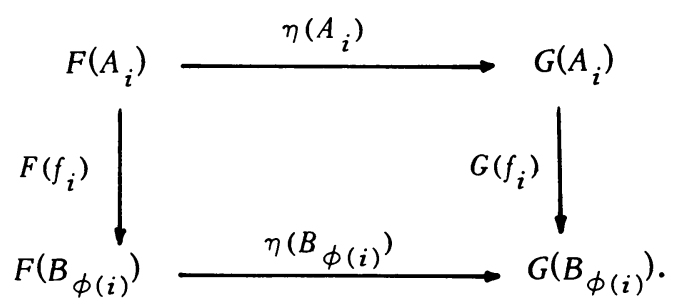

Taking direct limits, we get the commutative diagram

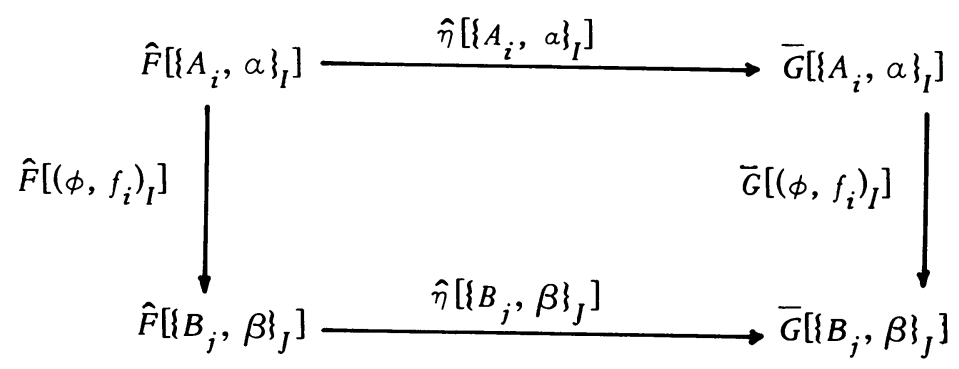

which verifies that $\hat{\eta}$ is natural.

If $\underline{A}$ is additive (cf. [12, pp. 65-67]), each of its hom sets has the structure of an abelian group with a distributive composition. Then in $\hat{A}$ each set $\underline{\hat{A}}\left(\left\{A_{i}\right\}_{I},\left\{B_{j}\right\}_{J}\right)=\varliminf_{\mathrm{lim}} \underline{\lim }_{J} \operatorname{Hom}_{A}\left(A_{i}, B_{j}\right)$ has an induced algebraic structure from $\underline{A}$, given as follows. If $\left(\phi^{1}, f_{i}^{1}\right)_{I}$ and $\left(\phi^{2}, f_{i}^{2}\right)_{I}$ are morphisms in $\underline{\hat{A}}\left(\left\{A_{i}\right\}_{I},\left\{B_{j}\right\}_{J}\right)$, we can use Lemma 3.2 to obtain a set map $\rho: I \rightarrow J$ and morphisms $\left(\rho, g_{i}^{1}\right)_{I} \sim$ $\left(\phi^{1}, f_{i}^{1}\right)_{I}$ and $\left(\rho, g_{i}^{2}\right)_{I} \sim\left(\phi^{2}, f_{i}^{2}\right)_{I}$. Then

$$
\left(\phi^{1}, f_{i}^{1}\right)_{I}+\left(\phi^{2}, f_{i}^{2}\right)_{I}=\left(\rho, g_{i}^{1}\right)_{I}+\left(\rho, g_{i}^{2}\right)_{I}=\left(\rho, g_{i}^{1}+g_{i}^{2}\right)_{I} .
$$

Associativity, commutativity and distributivity follow immediately. The zero morphism is $\left(\phi, 0_{i}\right)_{I}$ where $\phi: I \rightarrow J$ is any set map, and the inverse of $\left(\phi, f_{i}\right)_{I}$ is $\left(\phi,-f_{i}\right)_{I}$. Thus, each of the hom sets in $\underline{\hat{A}}$ has an induced abelian group structure with a distributive composition such that $I_{A}: \underline{A} \subseteq \underline{\hat{A}}$ is additive. If $F: \underline{A} \rightarrow \underline{B}$ is additive, then obviously so is $\hat{F}: \underline{A} \rightarrow \underline{B}$. The zero object of $\underline{A}$ is $\{0\}$. For the existence of biproducts in $\underline{\hat{A}}$, it is enough by Proposition 3.1 to consider objects $\left\{B_{i}^{1}\right\}_{I}$ and $\left\{B_{i}^{2}\right\}_{I}$ with the same directed set $I$. Since we have the commutative diagram 


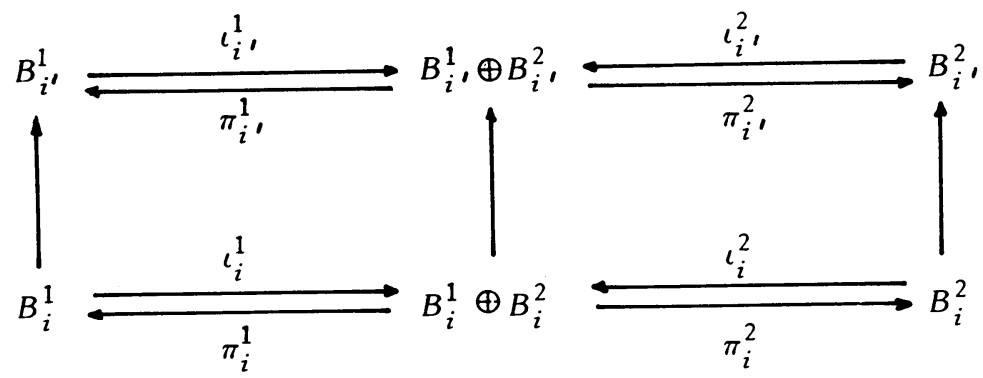

for $i \leq i^{\prime}$ in $I$, the biproduct of $\left\{B_{i}^{1}\right\}_{I}$ and $\left\{B_{i}^{2}\right\}_{I}$ and $\underline{\hat{A}}$ is given by

$$
\left\{B_{i}^{1}\right\}_{I} \underset{\left(\mathrm{id}, \pi_{i}^{1}\right)_{I}}{\stackrel{\left(\mathrm{id}, c_{i}^{1}\right)_{I}}{\rightleftarrows}}\left\{B_{i}^{1} \oplus B_{i}^{2}\right\}_{I} \stackrel{\left(\mathrm{id}, c_{i}^{2}\right)_{I}}{\stackrel{\left(\mathrm{id}, \pi_{i}^{2}\right)_{I}}{\rightleftarrows}}\left\{B_{i}^{2}\right\}_{I} .
$$

To prove (iv), we first show that, if $\underline{A}$ is abelian (cf. [5, pp. 35-36]), then so is $\hat{A}$. For the existence of kernels and cokernels, it is enough to consider morphisms (id, $\left.g_{i}\right)_{I}:\left\{B_{i}^{1}\right\}_{I} \rightarrow\left\{B_{i}^{2}\right\}_{I}$ satisfying (i) and (ii) of Corollary 3.6. For $i \leq i^{\prime}$ in $I$, we have the commutative diagram

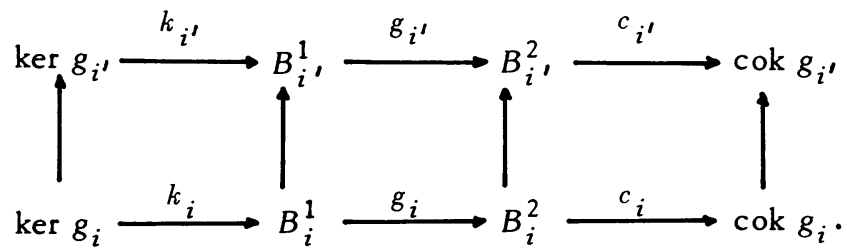

The kernel of (id, $\left.g_{i}\right)_{I}$ is given by

$$
\left\{\text { ker } g_{i}\right\} \stackrel{\left(\mathrm{id}, k_{i}\right)_{I}}{\longrightarrow}\left\{B_{i}^{1}\right\}_{I}
$$

and the cokernel by

$$
\left\{B_{i}^{2}\right\}_{I} \stackrel{\left(\mathrm{id}, c_{i}\right)_{I}}{\longrightarrow}\left\{\operatorname{cok} g_{i}\right\}_{I}
$$

It remains to prove the normality and conormality of $\underline{\hat{A}}$. For normality (conormality), it is sufficient to consider monomorphisms (epimorphisms) satisfying (i), (ii), and (iii) of Corollary 3.6. Then construct the cokernel (kernel) as above, and normality (conormality) is obvious. Note that $I_{A}: \underline{A} \hookrightarrow \underline{\hat{A}}$ is an exact functor.

For the AB5 property, we need to show that the directed direct limit of monomorphisms is a monomorphism. Let us be more precise. Any natural transformation $\eta$ between the functors $F, G: K \rightarrow \underline{A}$, where $K$ is a directed set, induces a unique morphism $\bar{\eta}: \lim _{K} F \rightarrow \lim _{K} G$ yielding the following commutative diagram of natural transformations: 


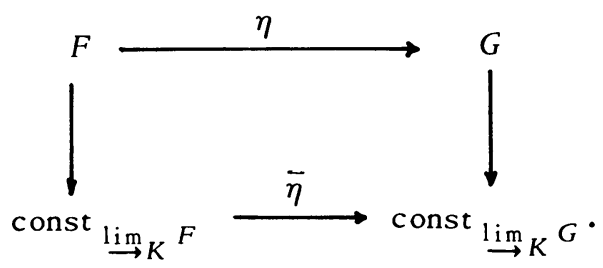

We want to show that, for each natural transformation $\eta$ with every $\eta(k), k \in K$, a monomorphism, $\bar{\eta}$ is a monomorphism (cf. [13, pp. 85-86]). But, it is clear that we can consider each $\eta(k): F(k) \rightarrow G(k)$ as a morphism (id, $\left.f_{i k}^{k}\right)_{I_{k}}:\left\{A^{k}\right\}_{I_{k}} \rightarrow\left\{B^{k}\right\}_{I_{k}}$ satisfying (i), (ii), and (iii) of Corollary 3.6, i.e. $f^{k}$ is a natural transformation with each $f_{i_{k}}^{k}, i_{k} \in I_{k}$, a monomorphism in $\underline{A}$. Recalling the construction of directed direct limits in $\hat{A}$ and using the fact that all the $f_{i_{k}}^{k}$ are monomorphisms, it is a straightforward verification that $\bar{\eta}$ is a monomorphism in $\underline{\hat{A}}$.

If $\underline{A}$ is small, then $\underline{\hat{A}}$ has the set of generators $\{\{A\}|A \in| \underline{A} \mid\}$, since every object in $\underline{A}$ is a directed direct limit of objects from this set. Thus, if $\underline{A}$ is small and abelian, $\underline{A}$ is an AB5 category with a set of generators. Then $\underline{A}$ has a generator and, hence, has enough injectives (cf. [7]). Q.E.D.

" $n "$ is, in fact, an adjoint functor (cf. [12, pp. 57-58]). Let Cat be the category whose objects consist of all categories and whose morphisms are all the functors between categories (cf. [10]). Let ind Cat be the subcategory of Cat consisting of categories with unique directed direct limits and functors which preserve directed direct limits. There is the embedding functor $E: \underline{\text { ind }} \underline{\text { Cat }} \hookrightarrow \underline{\text { Cat. }}$. Further-

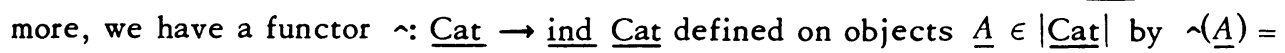
$\underline{\hat{A}}$ and on morphisms $F: \underline{A} \rightarrow \underline{B}$ in $\underline{\text { Cat by }} \wedge(F)=\left(\hat{I_{B}} \circ F\right)$.

Corollary 4.2. There is an adjunction $\eta$ : ind $\underline{\mathrm{Cat}}(\wedge(),) \rightarrow \underline{\mathrm{Cat}}(, E())$, i.e. - is an adjoint of $E$.

Proof. Define the adjunction $\eta$ by letting $\eta(\underline{A}, \underline{B})(F)=F \circ I_{\underline{A}}$ for $\underline{A} \in|\underline{\text { Cat }}|$, $\underline{B} \in|\underline{\text { ind }} \underline{\text { Cat }}|$, and $F \in \underline{\text { ind }} \underline{\text { Cat }}(\neg(\underline{A}), \underline{B})$. It is easily verified that $\eta$ is a natural isomorphism with inverse $\eta^{-1}$ : Cat $(, E()) \rightarrow \underline{\text { ind }}$ Cat $(\neg()$, ) given by $\eta^{-1}(\underline{A}, \underline{B})(G)=\hat{G}$ for $\underline{A} \in|\underline{\text { Cat }}|, \underline{B} \in|\underline{\text { ind }} \underline{\text { Cat }}|$, and $G \in \underline{\text { Cat }}(\underline{A}, E(\underline{B}))$. Q.E.D.

Remarks. (i) Recall that we defined the morphisms of $\underline{A}$ in $\$ 2$ so as to establish an equivalence $H$ between $\underline{\hat{A}}$ and the category of ind-representable functors in $\underline{\text { Ens }}{ }^{A}{ }^{\circ \mathrm{p}}$. When $\underline{A}$ is small and abelian, the ind-representable functors are precisely the left exact functors (cf. [14]), i.e. $\hat{A}$ is really the familiar functor category $\operatorname{Sex}\left(\underline{A}^{\mathrm{op}}, \underline{\mathrm{Ab}}\right)$ (cf. [6]).

(ii) The dual construction would yield a left completion $\breve{A}$ of $\underline{A}$ which is not only left complete, but also right complete with a projective generator, yielding a proof of the Exact Embedding Theorem and Mitchell's Embedding Theorem for abelian categories. 


\section{BIBLIOGRAPHY}

1. D. A. Buchsbaum, Exact categories and duality, Trans. Amer. Math. Soc. 80 (1955), 1-34. MR 17, 579 .

2. Satellites and universal functors, Ann. of Math. (2) 71 (1960), 199-209. MR $22 \# 3751$.

3. H. Cartan and S. Eilenberg, Homological algebra, Princeton Univ. Press, Princeton, N. J., 1956. MR 17, 1040.

4. P. Freyd, Functor categories and their application to relative homological algebra, University of Pennsylvania, Philadelphia, Pa., 1962 (mimeographed notes).

5. - Abelian categories. An introduction to the theory of functors, Harper's Series in Modern Math., Harper and Row, New York, 1964. MR 29 \#3517.

6. P. Gabriel, Des catégories Abéliennes, Bull. Soc. Math. France 90 (1962), 323-448. MR $38 \# 1144$.

7. A. Grothendieck, Sur quelques points d'algèbre homologique, Tôhoku Math. J. (2) 9 (1957), 119-221. MR $21 \# 1328$.

8. - Techniques de descente et théorèmes d'existence en géometrie algébrique (Extraits du Séminaire Bourbaki, 1957-1962), Éxposé 195, Secrétariat mathématique, Paris, 1962; reprint, Benjamin, New York, 1966. MR 33 \#5420g. \#A1301.

9. D. M. Kan, Adjoint functors, Trans. Amer. Math. Soc. 87 (1958), 294-329. MR 24

10. F. W. Lawvere, The category of categories as a foundation for mathematics, Proc. Conf. Categorical Algebra (La Jolla, Calif., 1965), Springer, New York, 1966, pp. 1-20. MR 34 \#7332.

11. S. Mac Lane, Homology, Die Grundlehren der math. Wissenschaften, Band 114, Academic Press, New York; Springer-Verlag, Berlin, 1963. MR 28 \#122. \#2053.

12. - Categorical algebra, Bull. Amer. Math. Soc. 71 (1965), 40-106. MR 30

13. B. Mitchell, Theory of categories, Pure and Appl. Math., vol. 17, Academic Press, New York, 1965. MR 34 \#2647.

14. H. B. Stauffer, A relationship between left exact and representable functors, Canad. J. Math. 23 (1971), 374-380. MR 42 \#6072.

15. F. Ulmer, Satelliten und derivierte Funktoren. I, Math. Z. 91 (1966), 216-266. MR 33 \#2705.

DEPARTMENT OF MATHEMATICS, UNIVERSITY OF CHICAGO, CHICAGO, ILLINOIS 60637

DEPARTMENT OF MATHEMATICS, UNIVERSITY OF BRITISH COLUMBIA, VANCOUVER, BRITISH COLUMBIA, CANADA

DEPARTMENT OF MATHEMATICS, CALIFORNIA STATE COLLEGE, HAYWARD, CALIFORNIA 94542 (Current address) 